\title{
Effect of Fertilizer Levels on Growth Attributes and Seed Yield of Lentil Varieties under Relay Cropping with Long Duration Rice in New Alluvial Zone of West Bengal, India
}

\author{
M.K. Kundu*, Sukanta Das, Sanjib Kumar Mandi and Rajib Nath \\ Department of Agronomy, Bidhan Chandra Krishi Viswavidyalaya, Mohanpur, Nadia, \\ West Bengal-741252, India \\ *Corresponding author
}

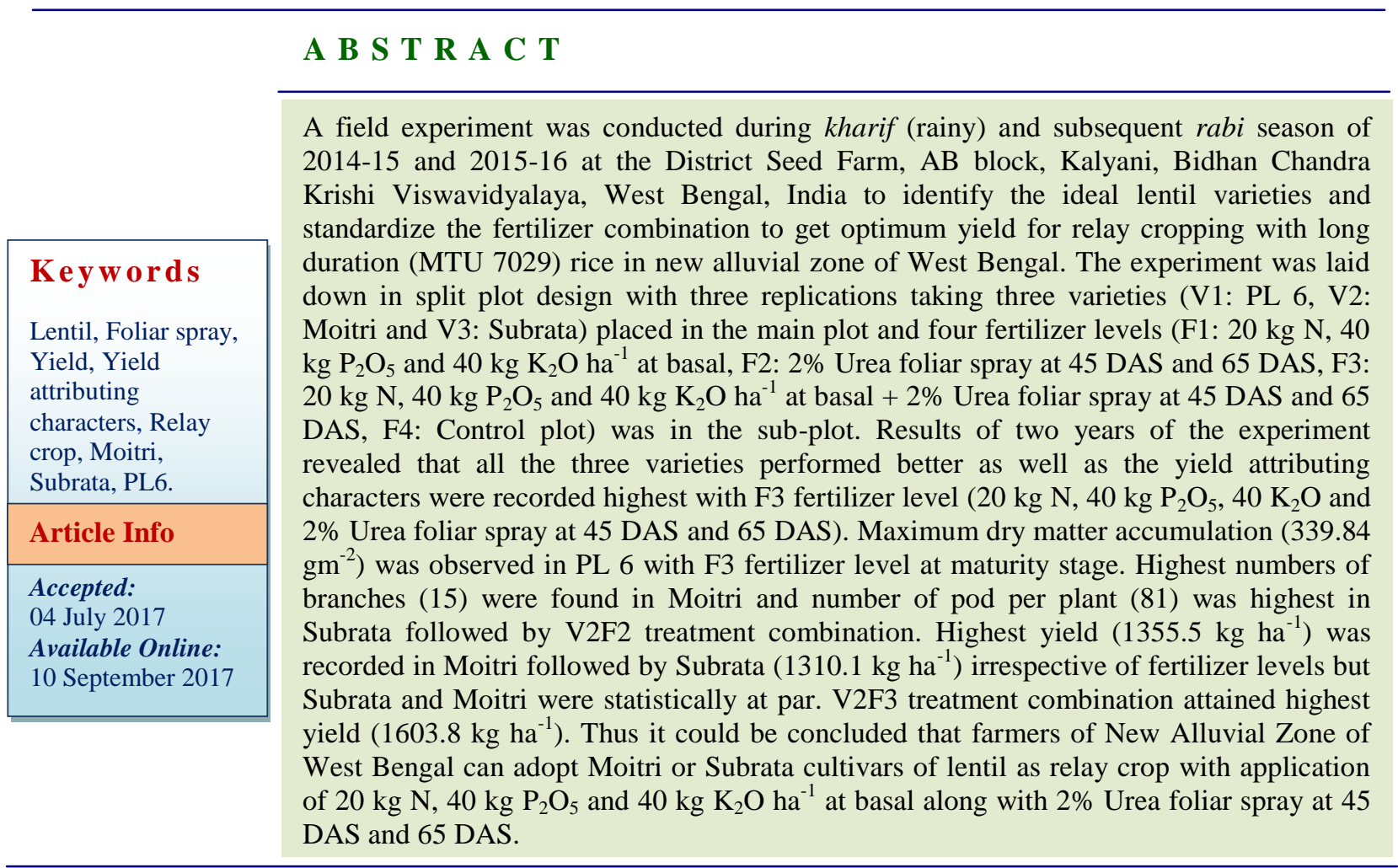

\section{Introduction}

The lentil (Lens culinaris Medikus subsp. culinaris) is a lens-shaped grain legume well known as a nutritious food. Lentil is a rich source of carbohydrate, protein, vitamins, minerals $(\mathrm{K}, \mathrm{P}, \mathrm{Fe}, \mathrm{Zn})$, dietary fibre with high energy value (de Almeida Costa et al., 2006) and it contain substantial amount of oleic, linoleic and palmitic acid (Roy et al., 2009). Lentil seeds are also a good source of essential minerals like calcium, phosphorous, iron and vitamin B, contains about $25 \%$ protein, $0.7 \%$ fat, $2.1 \%$ minerals, $0.7 \%$ fibre and $59 \%$ carbohydrate. It is used as a staple diet in many Middle Eastern countries and 
India. The flour can be mixed with cereals to make breads and cakes and as a food for infants (Williams and Singh 1988). Lentil, a cool season food legume ranks next only to chickpea in India. It is originated in the Near East and Mediterranean region (Dixit et al., 2009). The cultivated lentil is an annual long day plant. In some parts of this state it is grown as a paira crop. It is generally grown as a rainfed crop on marginal lands under residual soil moisture condition.

Relay cropping is one of the productive ways to increase the area of lentil or other pulse crops. Commonly relay cropping is known as Paira cropping in West Bengal or Utera cropping in Bihar and Chhattisgarh. The main objective of this type of cropping system is optimum utilization of residual moisture as well as residual fertility. This practice also helpful for getting proper time of sowing (Sharma et al., 2014). The aberrant onset and withdrawal of monsoons often poses problem in the land preparation of the winter crops (Parya et al., 2010). In West Bengal, lentil seeds are often broadcasted (as relay crop) in the standing crop of rice 15-20 days before harvesting to capitalize the residual moisture and ensure timely sowing as well as to get assured germination and skipping off the tillage operations during lentil growing.

However, the sowing of lentil often gets delayed due to weather variability and infestation of pest and diseases which lowers the yield (Ali et al., 2012). Late sown lentil faces terminal heat stress and drought during pod filling stage resulting in poor yield (Ali et al., 2012). Under such situation, early maturing cultivars with early vigour, fast vegetative growth, and quick canopy coverage may be successfully grown as relay crop in standing rice crop under no tillage condition. This further ensures conservation of natural resources, reduction of cost of production as there is no need of land preparation and other farm operations. The present experiment was carried out to identify the suitable lentil varieties with appropriate fertilizer dose for relay cropping after long duration (MTU 7029) rice. Generally lentil is sown as relay cropping in neglected condition. If care has been taken like cereal crops then there is a great potential to increase the yield of lentil with application of less amount of inputs. Care should be taken on view of optimum seed rate, appropriate variety, fertilizer dose etc.

The lower productivity of lentil could be attributed to cultivation in marginal and poor soils, inadequate irrigation, poor management practices, higher susceptibility to pest and disease and heavy flower drop. Among these, one of the important reasons for poor yields of lentils its fertilization aspect. Foliar spray of nutrients is the fastest way to boost up crop growth (Kuttimani, 2012).

Crop physiologists have recently developed the technique of foliar application to the agricultural crops (Kochhar and Krishnamoorthy, 1988; Smolen and Sady, 2008; Smolen and Sady, 2009). Foliar application is regarded as a preferred solution when the quick supply of nutrients is hindered or the soil conditions are not conducive for the absorption of nutrients (Salisbury and Ross, 1985). Most of the absorption by the young leaves takes place through the cuticles and hairs while some absorption might take place through stomata (Salisbury and Ross, 1985).

Foliar feeding can be an effective management tools to favourably influence pre-reproductive growth by compensating for environmentally induced stresses such as adverse growing conditions and/or poor nutrient availability. Early foliar application can make an already good crop better, either by stimulating more vigorous re-growth by 
increasing the yield potential. Foliar application should be timed to provide needed nutrients during the yield determining growth stages. Foliar application of nutrients could improve the nutrients utilization and lower environmental pollution through reducing the amounts of fertilizer added to soil (Abou- ElNour, 2002). Foliar feeding of a nutrient might have actually promoted root absorption of the same nutrient or other nutrients through improving root growth and increasing nutrients uptake (El-Fouly and El-Sayed, 1997). Foliar application of microelements is more beneficial than soil application. Since application rates are lesser as compare to soil application, same application could be obtained easily and crop reacts to nutrient application immediately (Zayed et al., 2011).

Keeping these in view, a study was undertaken with the following objectives:

To standardize the fertilizer doses to get optimum yield and

To identify the ideal lentil varieties suitable for relay cropping with long duration rice in New Alluvial Zone of West Bengal.

\section{Materials and Methods}

The investigation reported in the manuscript was carried out to study the effect of varieties and fertilizer levels on growth attributes and yield of lentil (Lens culinaris medikus) under relay cropping with long duration rice in new alluvial zone of West Bengal.

The field experiment was conducted at District Seed Farm, AB block, (22 ${ }^{\circ} 93^{\prime} \mathrm{N}$ latitude, $88^{\circ} 53^{\prime} \mathrm{E}$ longitude and $9.75 \mathrm{~m}$ above mean sea level) of Bidhan Chandra Krishi Viswavidyalaya, Kalyani, Nadia, West Bengal, India during Kharif season (July November) to rabi season (November March) of 2014-15 and 2015-16.
The experiment was conducted on a medium land, well-drained Gangetic alluvial soil (order: Inceptitisol), which belonged to the class of clayey loam with medium fertility and almost neutral in reaction. The field was generally kept under the cropping sequence of rice-lentil during last 5 years in kharif and rabi season respectively.

The on farm trial was conducted in split plot design with 3 replications. The plot size was gross $6 \mathrm{~m} . \times 2 \mathrm{~m}$. The treatments were 3 varieties of lentil (V1: PL 6, V2: Moitri and V3: Subrata) in main plots and four fertilizer levels (F1: $20 \mathrm{~kg} \mathrm{~N}, 40 \mathrm{~kg} \mathrm{P}_{2} \mathrm{O}_{5}$ and $40 \mathrm{~kg}$ $\mathrm{K}_{2} \mathrm{O}$ ha $^{-1}$ at basal, F2: $2 \%$ Urea foliar spray at 45 DAS and 65 DAS, F3: $20 \mathrm{~kg} \mathrm{~N}, 40 \mathrm{~kg}$ $\mathrm{P}_{2} \mathrm{O}_{5}$ and $40 \mathrm{~kg} \mathrm{~K}_{2} \mathrm{O}$ ha $^{-1}$ at basal $+2 \%$ Urea foliar spray at 45 DAS and 65 DAS, F4: Control plot) was in the sub-plot. Rice (Variety- MTU 7029) seedling was transplanted on 27.07.2014 and 22.07.2015 with $80 \mathrm{~kg} \mathrm{~N}, 40 \mathrm{~kg} \mathrm{P}_{2} \mathrm{O}_{5}$ and $40 \mathrm{~kg} \mathrm{~K}_{2} \mathrm{O}$ were applied with three splits doses of $\mathrm{N}\left(1 / 4^{\text {th }}, 1 / 2\right.$, $\left.1 / 4^{\text {th }}\right)$, full $\mathrm{P}_{2} \mathrm{O}_{5}$ and $\mathrm{K}_{2} \mathrm{O}$ at basal and harvested on 26.11.2014 and 23.11.2015. Lentil was sown on 11.11.2014 and 09.11.2015 during rabi (winter) season with $70 \mathrm{~kg} \mathrm{ha}^{-1}$ seed rate.

In each plot, 5 plants were randomly selected and tagged for repeated observations during the cropping period. The plant height was measured from ground level to the tip of plant canopy from five tagged plants in each plot at 45, 60, 75, 90 DAS and at harvest, then mean plant height was worked out.

For growth analysis, plant samples were collected at 45, 60, 75, 90 and 105 DAS. The roots were separated first and then the leaves, stems, branches, pods (at reproductive stage) were separated, which were kept in brown paper packets. They were dried separately in hot air oven at temperature of $80-90^{\circ} \mathrm{C}$ for 8 - 10 hours, till constant weights were 
obtained and converted $\mathrm{m}^{-2}$. After threshing, the seeds obtained from each plot were cleaned by removal of dry plant parts, dirt, other crop or weed seeds, etc. through winnowing and then it was dried under sun to achieve 12-13\% moisture content. The seed yield plot $^{-1}$ was transformed to $\mathrm{kg} \mathrm{ha}^{-1}$.

\section{Results and Discussion}

\section{Growth attributes}

Lentil is an important pulse crop which is a major protein resource to the people in India. Enhancing the productivity of lentil, to find out suitable cultivar and optimum fertilizer combination for relay cropping are important task of the researchers. In the present piece of work, the impact of cultivars and fertilizer levels of lentil regarding their growth and productivity has been discussed in the following paragraphs.

\section{Plant height}

The plant height recorded a continuous increase throughout the period of observation. In the first year, the plant height at the time of harvesting ranged from $53.70 \mathrm{~cm}$ to $62.92 \mathrm{~cm}$, whereas in second year it ranged from 53.66 $\mathrm{cm}$ to $60.81 \mathrm{~cm}$ among the cultivars irrespective of fertilizer levels.

Among the fertilizer levels the plant height in the first year ranged from $55.32 \mathrm{~cm}$ to 62.27 $\mathrm{cm}$ and in second year ranged from $55.93 \mathrm{~cm}$ to $60.84 \mathrm{~cm}$ irrespective of cultivars (Table 1a). Over interaction effect between cultivars and fertilizer levels plant height ranged from $50.3 \mathrm{~cm}$ to $70.3 \mathrm{~cm}$ and $49.0 \mathrm{~cm}$ to $64.9 \mathrm{~cm}$ in first year and second year respectively at the time of harvesting with significant variation among all the treatment combinations (Table 1b). At harvest there was no significant variation between Subrata and Moitri cultivars.
Pooled results of two years revealed that, the highest plant height was attained with PL 6 on 40 DAS $(25.9 \mathrm{~cm}), 55$ DAS $(34.3 \mathrm{~cm})$ and 85 DAS (49.5), Moitri attained highest plant height on 70 DAS $(42.8 \mathrm{~cm})$ among the cultivars irrespective of fertilizer levels. Among the fertilizer levels, irrespective of cultivars, maximum crop height was observed with F3 fertilizer levels at 40 DAS (25.79 $\mathrm{cm}), 55$ DAS $(34.72 \mathrm{~cm})$ as well as at the time of harvesting $(59.64 \mathrm{~cm})$. At F1 and F2 levels, maximum crop height was recorded on 85 DAS $(55.10 \mathrm{~cm})$ and on 70 DAS $(43.35 \mathrm{~cm})$ respectively. Plant height recorded continuous increase throughout the crop growth period with significant variation except at the time of harvesting and F1, F2 and F3 fertilizer levels were statistically at par throughout the crop growth period (Table 1c).

Impact of variety fertilizer on crop height was such that the maximum crop height was observed in V1F3 treatment combination on 40 DAS $(27.2 \mathrm{~cm})$ and 55 DAS $(36.0 \mathrm{~cm})$. Under F2 level, performance of Moitri was excellent on 70 DAS 70 DAS $(43.9 \mathrm{~cm})$. However the variety Subrata under F3 fertilizer level recorded height on 85 DAS $(60.6 \mathrm{~cm})$ and at harvest $(67.6 \mathrm{~cm})$ (Table 1d). The crop height in Moitri did not differ significantly because of variation in fertilizer levels at harvest. Similar result reported by Bhowmick, (2008).

\section{Dry matter accumulation}

Dry matter accumulation increased gradually with the advancement of crop age. Among the cultivars, total dry matter accumulation did not show significant variation in the both years. On 40 DAS PL 6 and Moitri recorded maximum dry matter accumulation in the first and second year respectively. The performance of Moitri on 55 and 70 DAS also was excellent with respect to dry matter accumulation. On 85 DAS the cultivar 
Subrata recorded maximum dry matter in the first $\left(176.8 \mathrm{~g} \mathrm{~m}^{-2}\right)$ and in the second year $\left(183.0 \mathrm{~g} \mathrm{~m}^{-2}\right)$. At harvest, the cultivar Subrata $\left(329.4 \mathrm{~g} \mathrm{~m}^{-2}\right)$ and PL $6\left(319.3 \mathrm{~g} \mathrm{~m}^{-2}\right)$ recorded maximum dry matter in the first and second year respectively (Table 2a).

Among the fertilizer levels dry matter accumulation increased progressively upto harvest irrespective of cultivars with significant effect except second year at 40 DAS. Maximum dry matter accumulation was recorded at F3 fertilizer level throughout the crop growth period except second year of 55 DAS. Dry matter accumulation recorded highest at 40 DAS $\left(17.4 \mathrm{~g} \mathrm{~m}^{-2}\right.$ and $\left.14.9 \mathrm{~g} \mathrm{~m}^{-2}\right)$, at 55 DAS $\left(47.3 \mathrm{~g} \mathrm{~m}^{-2}\right.$ and $\left.45.2 \mathrm{~g} \mathrm{~m}^{-2}\right)$ in F1 fertilizer level, at 70 DAS $\left(124.8 \mathrm{~g} \mathrm{~m}^{-2}\right.$ and $\left.117.9 \mathrm{~g} \mathrm{~m}^{-2}\right)$,at 85 DAS $\left(186.8 \mathrm{~g} \mathrm{~m}^{-2}\right.$ and $196.3 \mathrm{~g} \mathrm{~m}^{-2}$ ) and at the time of harvesting $\left(338.5 \mathrm{~g} \mathrm{~m}^{-2}\right.$ and332.1 $\left.\mathrm{g} \mathrm{m}^{-2}\right)$ in first year and second year respectively (Table $2 a$ ).

Highest dry matter accumulation observed at 40 DAS in V1F3 (19.6 $\left.\mathrm{g} \mathrm{m}^{-2}\right)$ treatment combination and V3F1 $\left(16.2 \mathrm{~g} \mathrm{~m}^{-2}\right)$ treatment combination in first year and second year respectively. On 55 DAS and 70 DAS maximum dry matter accumulation was observed in V3F3 treatment combination $\left(48.8 \mathrm{~g} \mathrm{~m}^{-2}\right.$ in first year and $50.5 \mathrm{~g} \mathrm{~m}^{-2}$ in second year and $126.6 \mathrm{~g} \mathrm{~m}^{-2}$ in first year and $118.5 \mathrm{~g} \mathrm{~m}^{-2}$ in second year respectively). At 85 DAS highest dry matter accumulation was observed at V1F3 $\left(212.4 \mathrm{~g} \mathrm{~m}^{-2}\right)$ and V2F3 $\left(202.9 \mathrm{~g} \mathrm{~m}^{-2}\right)$ treatment combination in first year and second year respectively. Highest dry matter accumulation was attained at harvest in V2F3 (346.5 $\left.\mathrm{g} \mathrm{m}^{-2}\right)$ and V1F3 $\left(345.2 \mathrm{~g} \mathrm{~m}^{-2}\right)$ treatment combination in first year and second year respectively (Table $2 b$ ).

Pooled analysis revealed that, among the cultivars total dry matter accumulation exhibited no significant variations throughout the crop growing period irrespective of fertilizer levels. Highest dry matter accumulation was observe in PL $6\left(15.32 \mathrm{~g} \mathrm{~m}^{-2}\right.$ ) followed by Moitri (15.02 $\mathrm{g} \mathrm{m}^{-2}$ ) and Moitri $\left(42.84 \mathrm{~g} \mathrm{~m}^{-2}\right)$ followed by Subrata (42.02 $\mathrm{g} \mathrm{m}^{-2}$ ) at 40 DAS and 55 DAS respectively but at 70 DAS, 85 DAS and at harvest recorded highest dry matter accumulation in Subrata (109.34 g $\mathrm{m}^{-2}, \quad 179.88 \mathrm{~g} \mathrm{~m}^{-2}$, and $319.18 \mathrm{~g} \mathrm{~m}^{-2}$ respectively) followed by Moitri $108.86 \mathrm{~g} \mathrm{~m}^{-2}$, $177.12 \mathrm{~g} \mathrm{~m}^{-2}$ and $317.68 \mathrm{~g} \mathrm{~m}^{-2}$ at 70 DAS, 85 DAS and at harvest respectively (Table $2 \mathrm{c}$ ).

Among the fertilizer levels irrespective of cultivars dry matter accumulation increased progressively upto harvest with significant variation. Throughout the crop growth period highest dry matter accumulation was observed in F3 fertilizer levels. Dry matter accumulation was $16.12 \mathrm{~g} \mathrm{~m}^{-2}, 46.95 \mathrm{~g} \mathrm{~m}^{-2}$, $121.35 \mathrm{~g} \mathrm{~m}^{-2}, 196.57 \mathrm{~g} \mathrm{~m}^{-2}$ and $335.30 \mathrm{~g} \mathrm{~m}^{-2}$ in 40 DAS, 55 DAS, 70 DAS, and 85 DAS and at harvest respectively. At 40 DAS F1, F2 and F3 fertilizer levels were statistically at par. The percentage increase in dry matter accumulation was 5.3, 2.9 and 10.8 for F1, F2 and $\mathrm{F} 3$ respectively over control (Table $2 \mathrm{c}$ ).

The interaction effect was significant throughout the growth period. Highest dry matter accumulation was attained in V1F3 $\left(17.54 \mathrm{~g} \mathrm{~m}^{-2}\right)$ followed by V3F1 (16.88 $\left.\mathrm{gm}^{-2}\right)$ treatment combination at 40 DAS. All treatment combinations except V1F4, V2F4, V3F2 and V3F4 were statistically at par at 40 DAS. At 55 DAS maximum dry matter accumulation recorded with V3F3 (49.65 $\left.\mathrm{g} \mathrm{m}^{-2}\right)$ followed by V1F4 (48.03 $\left.\mathrm{g} \mathrm{m}^{-2}\right)$ treatment combination and there was no significant variation between $\mathrm{V} 1 \mathrm{~F} 3, \mathrm{~V} 1 \mathrm{~F} 4, \mathrm{~V} 2 \mathrm{~F} 1, \mathrm{~V} 2 \mathrm{~F} 3$ and V3F3 treatment combinations. At 70 DAS maximum dry matter was accumulated in V3F3 (122.54 $\mathrm{g} \mathrm{m}^{-2}$ ) followed by V2F3 $\left(121.4 \mathrm{~g} \mathrm{~m}^{-2}\right)$ treatment combination where $\mathrm{V} 1 \mathrm{~F} 4, \mathrm{~V} 2 \mathrm{~F} 3, \mathrm{~V} 3 \mathrm{~F} 2$ and $\mathrm{V} 3 \mathrm{~F} 3$ were statistically at par. 
Table.1a Plant height $(\mathrm{cm})$ of lentil as influenced by cultivar and fertilizer level

\begin{tabular}{|c|c|c|c|c|c|c|c|c|c|c|}
\hline \multirow{2}{*}{ Treatment } & \multicolumn{2}{|c|}{40 DAS } & \multicolumn{2}{|c|}{55 DAS } & \multicolumn{2}{|c|}{70 DAS } & \multicolumn{2}{|c|}{85 DAS } & \multicolumn{2}{|c|}{ At Harvest } \\
\hline & $1^{\text {st }}$ year & $2^{\text {nd }}$ year & $1^{\text {st }}$ year & $2^{\text {nd }}$ year & $1^{\text {st }}$ year & $2^{\text {nd }}$ year & $1^{\text {st }}$ year & $2^{\text {nd }}$ year & $1^{\text {st }}$ year & $2^{\text {nd }}$ year \\
\hline & \multicolumn{10}{|c|}{ Cultivars } \\
\hline V1 & 25.21 & 26.76 & 34.76 & 33.97 & 41.89 & 42.05 & 50.47 & 48.57 & 53.70 & 53.66 \\
\hline $\mathrm{V} 2$ & 22.97 & 25.82 & 33.52 & 33.79 & 43.81 & 41.93 & 60.33 & 48.91 & 62.00 & 57.81 \\
\hline V3 & 24.07 & 23.47 & 32.74 & 32.42 & 42.87 & 40.10 & 60.59 & 49.33 & 62.92 & 60.81 \\
\hline \multirow[t]{2}{*}{$\mathrm{CD}$ at $5 \%$} & NS & $\mathrm{NS}$ & NS & NS & NS & NS & 8.0 & $\mathrm{NS}$ & 7.8 & NS \\
\hline & \multicolumn{10}{|c|}{ Fertilizer combination } \\
\hline $\mathrm{F} 1$ & 24.43 & 24.67 & 33.83 & 32.51 & 44.38 & 41.04 & 60.33 & 49.88 & 62.27 & 55.93 \\
\hline $\mathrm{F} 2$ & 23.93 & 27.46 & 33.32 & 36.03 & 43.10 & 43.60 & 59.62 & 49.48 & 62.11 & 56.71 \\
\hline F3 & 25.47 & 26.11 & 35.06 & 34.39 & 43.37 & 41.77 & 55.91 & 50.00 & 58.44 & 60.84 \\
\hline $\mathrm{F} 4$ & 22.51 & 23.16 & 32.48 & 30.64 & 40.59 & 39.02 & 52.66 & 46.39 & 55.33 & 56.22 \\
\hline $\mathrm{CD}$ at $5 \%$ & 1.9 & 2.9 & NS & 2.6 & NS & 2.7 & NS & NS & NS & NS \\
\hline
\end{tabular}

Table.1b Interaction effect (cultivar $\times$ fertilizer level) on plant height $(\mathrm{cm})$ of lentil

\begin{tabular}{|c|c|c|c|c|c|c|c|c|c|c|}
\hline V1F1 & 27.0 & 23.7 & 32.9 & 33.5 & 40.4 & 43.8 & 53.9 & 50.1 & 56.1 & 49.0 \\
\hline V1F2 & 23.5 & 28.3 & 34.0 & 34.8 & 42.3 & 42.7 & 51.5 & 48.5 & 55.0 & 50.3 \\
\hline V1F3 & 25.6 & 28.8 & 37.5 & 34.6 & 44.9 & 39.5 & 49.1 & 48.2 & 53.3 & 62.7 \\
\hline V1F4 & 24.8 & 26.2 & 34.6 & 32.9 & 39.9 & 42.1 & 47.4 & 47.5 & 50.3 & 52.7 \\
\hline V2F1 & 21.4 & 25.0 & 33.8 & 32.0 & 46.3 & 40.4 & 62.4 & 48.6 & 64.0 & 58.6 \\
\hline V2F2 & 24.0 & 27.4 & 32.9 & 37.0 & 43.7 & 44.3 & 66.1 & 49.9 & 67.7 & 61.6 \\
\hline V2F3 & 26.7 & 26.0 & 35.3 & 32.8 & 41.8 & 42.5 & 49.3 & 49.7 & 51.7 & 55.0 \\
\hline V2F4 & 19.8 & 24.8 & 32.0 & 33.4 & 43.5 & 40.6 & 63.5 & 47.4 & 64.7 & 56.0 \\
\hline V3F1 & 24.8 & 25.3 & 34.7 & 32.0 & 46.4 & 39.0 & 64.7 & 50.9 & 66.7 & 60.2 \\
\hline V3F2 & 24.4 & 26.6 & 33.2 & 36.3 & 43.3 & 43.8 & 61.3 & 50.0 & 63.7 & 58.2 \\
\hline V3F3 & 24.1 & 23.5 & 32.4 & 35.8 & 43.5 & 43.2 & 69.2 & 52.1 & 70.3 & 64.9 \\
\hline V3F4 & 23.0 & 18.5 & 30.8 & 25.7 & 38.4 & 34.4 & 47.1 & 44.2 & 51.0 & 60.0 \\
\hline $\begin{array}{l}\mathbf{F} \times \mathbf{V} \\
\mathrm{CD} \text { at } 5 \% \\
\mathbf{V} \times \mathbf{F}\end{array}$ & 3.8 & NS & NS & 5.1 & NS & 5.3 & 13.7 & NS & 12.1 & 8.4 \\
\hline $\mathrm{CD}$ at $5 \%$ & 4.2 & NS & NS & 5.8 & NS & 6.1 & 13.5 & NS & 12.0 & 8.7 \\
\hline
\end{tabular}

Where, V1: PL6, V2: Moitri, V3: Subrata, F1: Apply $20 \mathrm{~kg}, 40 \mathrm{~kg}$ and $40 \mathrm{~kg} \mathrm{~N}, \mathrm{P}_{2} \mathrm{O}_{5}$ and $\mathrm{K}_{2} \mathrm{O}$ respectively as basal, F2: $2 \%$ foliar spray at 45 DAS and 65 DAS, F3: Apply $20 \mathrm{~kg}, 40 \mathrm{~kg}$ and $40 \mathrm{~kg} \mathrm{~N}, \mathrm{P}_{2} \mathrm{O}_{5}$ and $\mathrm{K}_{2} \mathrm{O}$ respectively as basal $+2 \%$ foliar spray at $45 \mathrm{DAS}$ and 65 DAS, F4: Control plot and NS: non-significant. 
Table.1c Plant height (cm) of lentil as influenced by cultivar and fertilizer level (pooled data of two years)

\begin{tabular}{|c|c|c|c|c|c|}
\hline Treatment & $\begin{array}{l}40 \text { DAS } \\
\text { Pooled }\end{array}$ & $\begin{array}{l}55 \text { DAS } \\
\text { Pooled }\end{array}$ & $\begin{array}{l}70 \text { DAS } \\
\text { Pooled }\end{array}$ & $\begin{array}{c}85 \text { DAS } \\
\text { Pooled }\end{array}$ & $\begin{array}{c}\text { At Harvest } \\
\text { Pooled }\end{array}$ \\
\hline & \multicolumn{5}{|c|}{ Cultivars } \\
\hline V1 & 25.99 & 34.36 & 41.97 & 49.52 & 53.68 \\
\hline $\mathrm{V} 2$ & 24.39 & 33.65 & 42.87 & 44.62 & 59.90 \\
\hline V3 & 23.76 & 32.58 & 41.48 & 45.96 & 61.86 \\
\hline \multirow{2}{*}{$\mathrm{CD}$ at $5 \%$} & NS & NS & NS & 3.62 & 3.78 \\
\hline & \multicolumn{5}{|c|}{ Fertilizer combination } \\
\hline F1 & 24.55 & 33.16 & 42.71 & 55.10 & 59.10 \\
\hline $\mathrm{F} 2$ & 25.70 & 34.67 & 43.35 & 54.55 & 59.41 \\
\hline F3 & 25.79 & 34.72 & 42.57 & 52.95 & 59.64 \\
\hline $\mathrm{F} 4$ & 23.83 & 31.56 & 39.81 & 49.52 & 55.78 \\
\hline $\mathrm{CD}$ at $5 \%$ & 1.3 & 1.9 & 2.3 & 3.9 & $\mathrm{NS}$ \\
\hline
\end{tabular}

Table.1d Interaction effect (cultivar $\times$ fertilizer level) on plant height $(\mathrm{cm})$ of lentil (pooled data of two years)

\begin{tabular}{|c|c|c|c|c|c|}
\hline V1F1 & 25.38 & 33.24 & 42.12 & 41.97 & 52.55 \\
\hline V1F2 & 25.89 & 34.39 & 42.53 & 49.99 & 52.63 \\
\hline V1F3 & 27.20 & 36.05 & 42.19 & 48.65 & 58.01 \\
\hline V1F4 & 25.47 & 33.76 & 41.02 & 47.46 & 51.61 \\
\hline V2F1 & 23.20 & 32.94 & 43.33 & 55.51 & 61.32 \\
\hline V2F2 & 25.71 & 34.93 & 43.97 & 57.98 & 64.65 \\
\hline V2F3 & 26.36 & 34.04 & 42.16 & 49.52 & 53.32 \\
\hline V2F4 & 22.29 & 32.69 & 42.02 & 55.45 & 60.32 \\
\hline V3F1 & 25.05 & 33.33 & 42.67 & 57.82 & 53.41 \\
\hline V3F2 & 25.48 & 34.70 & 43.59 & 55.67 & 60.94 \\
\hline V3F3 & 23.80 & 34.70 & 43.35 & 60.68 & 67.60 \\
\hline V3F4 & 20.72 & 28.21 & 36.37 & 45.66 & 55.50 \\
\hline $\mathbf{V} \times \mathbf{F}(\mathrm{CD}$ at $5 \%)$ & NS & $\mathrm{NS}$ & NS & 6.84 & 6.47 \\
\hline
\end{tabular}

Where, V1: PL6, V2: Moitri, V3: Subrata, F1: Apply $20 \mathrm{~kg}, 40 \mathrm{~kg}$ and $40 \mathrm{~kg} \mathrm{~N}, \mathrm{P}_{2} \mathrm{O}_{5}$ and $\mathrm{K}_{2} \mathrm{O}$ respectively as basal, F2: $2 \%$ foliar spray at 45 DAS and 65 DAS, F3: Apply $20 \mathrm{~kg}, 40 \mathrm{~kg}$ and $40 \mathrm{~kg} \mathrm{~N}, \mathrm{P}_{2} \mathrm{O}_{5}$ and $\mathrm{K}_{2} \mathrm{O}$ respectively as basal + $2 \%$ foliar spray at $45 \mathrm{DAS}$ and $65 \mathrm{DAS}$, F4: Control plot and NS: non-significant. 
Table.2a Dry matter accumulation $\left(\mathrm{g} \mathrm{m}^{-2}\right)$ of Lentil as influenced by cultivar and fertilizer level

\begin{tabular}{|c|c|c|c|c|c|c|c|c|c|c|}
\hline \multirow[b]{2}{*}{ Treatment } & \multicolumn{2}{|c|}{40 DAS } & \multicolumn{2}{|c|}{55 DAS } & \multicolumn{2}{|c|}{70 DAS } & \multicolumn{2}{|c|}{85 DAS } & \multicolumn{2}{|c|}{ At Harvest } \\
\hline & $\begin{array}{c}1^{\text {st }} \\
\text { year }\end{array}$ & $2^{\text {nd }}$ year & $1^{\text {st }}$ year & $2^{\text {nd }}$ year & $1^{\text {st }}$ year & $2^{\text {nd }}$ year & $1^{\text {st }}$ year & $2^{\text {nd }}$ year & $1^{\text {st }}$ year & $2^{\text {nd }}$ year \\
\hline & \multicolumn{10}{|c|}{ Cultivars } \\
\hline V1 & 16.0 & 14.6 & 40.9 & 41.2 & 106.5 & 109.0 & 168.8 & 176.4 & 313.3 & 319.3 \\
\hline $\mathrm{V} 2$ & 15.3 & 14.7 & 43.5 & 42.2 & 108.9 & 118.1 & 176.1 & 178.1 & 323.3 & 312.1 \\
\hline V3 & 15.0 & 14.4 & 43.3 & 40.8 & 107.6 & 111.0 & 176.8 & 183.0 & 329.4 & 319.0 \\
\hline \multirow[t]{2}{*}{$\mathrm{CD}$ at $5 \%$} & NS & $\mathrm{NS}$ & NS & $\mathrm{NS}$ & NS & $\mathrm{NS}$ & NS & NS & NS & NS \\
\hline & \multicolumn{10}{|c|}{ Fertilizer combination } \\
\hline F1 & 16.6 & 14.8 & 42.3 & 45.2 & 101.7 & 111.0 & 178.3 & 180.3 & 327.6 & 319.7 \\
\hline $\mathrm{F} 2$ & 15.0 & 14.9 & 44.3 & 38.7 & 117.7 & 106.7 & 174.1 & 184.2 & 321.3 & 311.9 \\
\hline F3 & 17.4 & 14.9 & 47.3 & 44.6 & 124.8 & 117.9 & 186.8 & 196.3 & 338.5 & 332.1 \\
\hline $\mathrm{F} 4$ & 12.8 & 13.8 & 36.4 & 38.0 & 86.6 & 103.0 & 146.3 & 165.9 & 307.1 & 298.1 \\
\hline $\mathrm{CD}$ at $5 \%$ & 2.4 & NS & 6.1 & 4.5 & 9.4 & 7.0 & 18.2 & 14 & 16.5 & 21.1 \\
\hline
\end{tabular}

Table.2b Interaction effect (cultivar $\times$ fertilizer level) on dry matter accumulation $\left(\mathrm{g} \mathrm{m}^{-2}\right)$ of lentil

\begin{tabular}{c|cccccccccc}
\hline V1F1 & 16.0 & 13.9 & 38.7 & 38.4 & 93.8 & 110.7 & 191.7 & 178.6 & 314.9 & 306.9 \\
V1F2 & 15.1 & 15.0 & 41.6 & 41.0 & 114.4 & 107.3 & 152.2 & 168.6 & 307.5 & 312.1 \\
V1F3 & 19.6 & 15.4 & 44.5 & 46.5 & 124.4 & 115.8 & 212.4 & 185.3 & 334.5 & 345.2 \\
V1F4 & 13.4 & 14.1 & 38.6 & 38.9 & 93.5 & 102.4 & 119.2 & 173.0 & 296.1 & 296.9 \\
V2F1 & 16.1 & 14.4 & 46.8 & 49.2 & 102.5 & 110.9 & 164.7 & 191.1 & 315.4 & 318.8 \\
V2F2 & 15.2 & 15.7 & 44.0 & 39.7 & 118.9 & 105.0 & 183.7 & 158.3 & 315.9 & 302.4 \\
V2F3 & 16.1 & 14.8 & 48.6 & 42.9 & 123.4 & 119.4 & 187.4 & 202.9 & 346.5 & 326.6 \\
V2F4 & 13.8 & 14.1 & 34.7 & 36.9 & 90.5 & 100.2 & 168.7 & 160.3 & 315.3 & 300.6 \\
V3F1 & 17.6 & 16.2 & 41.3 & 41.9 & 108.7 & 111.3 & 178.6 & 171.2 & 322.5 & 333.6 \\
V3F2 & 14.9 & 14.0 & 47.3 & 35.5 & 119.7 & 107.8 & 186.6 & 195.5 & 310.7 & 321.1 \\
V3F3 & 16.3 & 14.3 & 48.8 & 50.5 & 126.6 & 118.5 & 190.7 & 200.8 & 334.5 & 324.5 \\
V3F4 & 11.2 & 13.2 & 35.8 & 35.3 & 75.6 & 106.5 & 151.1 & 164.4 & 309.9 & 296.7 \\
\hline F $\times$ VCD at 5\% & NS & NS & NS & 9.1 & NS & NS & 36.2 & 26.5 & NS & NS \\
V $\times$ FCD at 5\% & NS & NS & NS & 11.2 & NS & NS & 41.2 & 26.9 & NS & NS \\
\hline
\end{tabular}

Where, V1: PL6, V2: Moitri, V3: Subrata, F1: Apply $20 \mathrm{~kg}, 40 \mathrm{~kg}$ and $40 \mathrm{~kg} \mathrm{~N}, \mathrm{P}_{2} \mathrm{O}_{5}$ and $\mathrm{K}_{2} \mathrm{O}$ respectively as basal, F2: $2 \%$ foliar spray at 45 DAS and 65 DAS, F3: Apply $20 \mathrm{~kg}, 40 \mathrm{~kg}$ and $40 \mathrm{~kg} \mathrm{~N}, \mathrm{P}_{2} \mathrm{O}_{5}$ and $\mathrm{K}_{2} \mathrm{O}$ respectively as basal $+2 \%$ foliar spray at 45 DAS and 65 DAS,

F4: Control plot and NS: non-significant. 
Table.2c Dry matter accumulation $\left(\mathrm{g} \mathrm{m}^{-2}\right)$ of lentil as influenced by cultivar and fertilizer level (pooled data of two years)

\begin{tabular}{c|ccccc}
\hline Treatment & $\begin{array}{c}\text { 40 DAS } \\
\text { Pooled }\end{array}$ & $\begin{array}{c}\text { 55 DAS } \\
\text { Pooled }\end{array}$ & $\begin{array}{c}\text { 70 DAS } \\
\text { Pooled }\end{array}$ & $\begin{array}{c}\text { 85 DAS } \\
\text { Pooled }\end{array}$ & $\begin{array}{c}\text { At Harvest } \\
\text { Pooled }\end{array}$ \\
\hline & & Cultivars & \\
\hline V1 & 15.32 & 41.03 & 107.78 & 172.62 & 314.27 \\
V2 & 15.02 & 42.84 & 108.86 & 177.12 & 317.68 \\
V3 & 14.72 & 42.04 & 109.34 & 179.88 & 319.18 \\
\hline CD at 5\% & 1.69 & 4.15 & 4.42 & 14.61 \\
\hline & \multicolumn{5}{c}{ Fertilizer combination } \\
\hline F1 & 15.70 & 42.71 & 106.32 & 179.32 & 311.687 \\
F2 & 14.99 & 41.51 & 112.17 & 196.57 & 335.30 \\
F3 & 16.12 & 46.95 & 121.35 & 156.10 & 302.58 \\
\hline F4 & 13.30 & 36.70 & 94.80 & 11.00 & 12.83 \\
\hline
\end{tabular}

Table.2d Interaction effect (cultivar $\times$ fertilizer level) on dry matter accumulation $\left(\mathrm{g} \mathrm{m}^{-2}\right)$ of lentil (pooled data of two years)

\begin{tabular}{|c|c|c|c|c|c|}
\hline V1F1 & 14.97 & 38.53 & 102.24 & 185.16 & 310.93 \\
\hline V1F2 & 15.05 & 41.33 & 110.82 & 160.41 & 309.82 \\
\hline V1F3 & 17.54 & 45.48 & 120.12 & 198.82 & 339.84 \\
\hline V1F4 & 13.74 & 48.77 & 97.95 & 146.07 & 296.51 \\
\hline V2F1 & 15.24 & 48.03 & 106.71 & 177.87 & 317.09 \\
\hline V2F2 & 15.45 & 41.83 & 111.95 & 170.99 & 309.13 \\
\hline V2F3 & 15.46 & 45.71 & 121.40 & 195.15 & 336.55 \\
\hline V2F4 & 13.94 & 35.78 & 95.38 & 164.48 & 307.95 \\
\hline V3F1 & 16.88 & 41.58 & 109.99 & 174.93 & 328.03 \\
\hline V3F2 & 14.47 & 41.38 & 113.75 & 191.07 & 315.89 \\
\hline V3F3 & 15.34 & 49.65 & 122.54 & 195.75 & 329.52 \\
\hline V3F4 & 12.19 & 35.55 & 91.06 & 157.75 & 303.28 \\
\hline $\mathbf{V} \times \mathbf{F ~ C D}$ at $5 \%$ & 2.72 & 6.24 & 9.75 & 19.06 & 22.23 \\
\hline
\end{tabular}

Where, V1: PL6, V2: Moitri, V3: Subrata, F1: Apply $20 \mathrm{~kg}, 40 \mathrm{~kg}$ and $40 \mathrm{~kg} \mathrm{~N}, \mathrm{P}_{2} \mathrm{O}_{5}$ and $\mathrm{K}_{2} \mathrm{O}$ respectively as basal, F2: $2 \%$ foliar spray at 45 DAS and 65 DAS, F3: Apply $20 \mathrm{~kg}, 40 \mathrm{~kg}$ and $40 \mathrm{~kg} \mathrm{~N}, \mathrm{P}_{2} \mathrm{O}_{5}$ and $\mathrm{K}_{2} \mathrm{O}$ respectively as basal $+2 \%$ foliar spray at 45 DAS and 65 DAS, F4: Control plot and NS: non-significant. 
Table.3a Yield $\left(\mathrm{kg} \mathrm{ha}^{-1}\right)$ of lentil as influenced by cultivar and fertilizer level

\begin{tabular}{|c|c|c|c|}
\hline \multirow[b]{2}{*}{ Treatment } & \multicolumn{3}{|c|}{ Yield $\left(\mathrm{kg} \mathrm{ha}^{-1}\right)$} \\
\hline & $1^{\text {st }}$ Year (2014 -2015) & $\operatorname{lr}(2015-2016)$ & Pooled \\
\hline & \multicolumn{3}{|c|}{ Cultivars } \\
\hline V1 & 1377.4 & 1174.7 & 1276.1 \\
\hline $\mathrm{V} 2$ & 1519.4 & 1191.5 & 1355.5 \\
\hline V3 & 1319.8 & 1301.8 & 1310.1 \\
\hline \multirow[t]{2}{*}{$\mathrm{CD}$ at $5 \%$} & 109.7 & 51.7 & 49.0 \\
\hline & \multicolumn{3}{|c|}{ Fertilizer combination } \\
\hline $\mathrm{F} 1$ & 1467.9 & 1328.2 & 1398.0 \\
\hline $\mathrm{F} 2$ & 1386.4 & 1198.7 & 1292.5 \\
\hline $\mathrm{F} 3$ & 1670.4 & 1448.0 & 1559.2 \\
\hline $\mathrm{F} 4$ & 1097.6 & 915.8 & 1006.6 \\
\hline $\mathrm{CD}$ at $5 \%$ & 109.4 & 45.0 & 56.7 \\
\hline
\end{tabular}

Table.3b Interaction effect (cultivar $\times$ fertilizer level) on yield $\left(\mathrm{kg} \mathrm{ha}^{-1}\right)$ of lentil

\begin{tabular}{|c|c|c|c|}
\hline V1F1 & 1545.2 & 1319.5 & 1432.3 \\
\hline V1F2 & 1211.3 & 1156.3 & 1181.8 \\
\hline V1F3 & 1722.0 & 1389.2 & 1555.6 \\
\hline V1F4 & 1031.3 & 833.9 & 932.5 \\
\hline V2F1 & 1502.7 & 1256.7 & 1379.7 \\
\hline V2F2 & 1578.7 & 1134.4 & 1356.5 \\
\hline V2F3 & 1697.7 & 1509.9 & 1603.8 \\
\hline V2F4 & 1298.8 & 864.8 & 1081.8 \\
\hline V3F1 & 1355.9 & 1408.3 & 1382.1 \\
\hline V3F2 & 1369.2 & 1305.2 & 1337.2 \\
\hline V3F3 & 1591.7 & 1444.9 & 1518.3 \\
\hline V3F4 & 962.6 & 1048.7 & 1005.6 \\
\hline \multirow{3}{*}{$\mathrm{CD}$ at $5 \%$} & \multicolumn{2}{|c|}{$F \times V$} & \\
\hline & 202.6 & 84.7 & 98.2 \\
\hline & \multicolumn{2}{|c|}{$V \times F$} & \\
\hline $\mathrm{CD}$ at $5 \%$ & 195.9 & 84.3 & \\
\hline
\end{tabular}

Where, V1: PL6, V2: Moitri, V3: Subrata, F1: Apply $20 \mathrm{~kg}, 40 \mathrm{~kg}$ and $40 \mathrm{~kg} \mathrm{~N}, \mathrm{P}_{2} \mathrm{O}_{5}$ and $\mathrm{K}_{2} \mathrm{O}$ respectively as basal, F2: $2 \%$ foliar spray at 45 DAS and 65 DAS, F3: Apply $20 \mathrm{~kg}, 40 \mathrm{~kg}$ and $40 \mathrm{~kg} \mathrm{~N}, \mathrm{P}_{2} \mathrm{O}_{5}$ and $\mathrm{K}_{2} \mathrm{O}$ respectively as basal $+2 \%$ foliar spray at 45 DAS and 65 DAS, F4: Control plot and NS: non-significant. 
At 85 DAS highest dry matter accumulation attained in V1F3 (198.82 $\left.\mathrm{g} \mathrm{m}^{-2}\right)$ followed by V3F3 (195.75 $\mathrm{g} \mathrm{m}^{-2}$ ) followed by Moitri $\left(108.86 \mathrm{~g} \mathrm{~m}^{-2}\right)$ treatment combination and no significant variation was observed between V1F1, V3F3, V2F3, V3F2 and V3F3 treatment combinations. At the time of harvesting maximum dry matter accumulated in V1F3 followed by V2F3 (336.55 followed by Moitri $\left(108.86 \mathrm{~g} \quad \mathrm{~m}^{-2}\right)$ treatment combination and there was no significant variation found between V1F3, V2F3, V3F1 and V3F3 treatment combinations (Table 2d).

Martens et al., (2001) reported that average dry matter accumulation of lentil in relay cropping was $634 \mathrm{~kg} \mathrm{ha}^{-1}$.

\section{Seed yield}

The varietal response, irrespective of fertilizer levels significantly differed with respect to the seed yield of crop. In first year Moitri $\left(1519.4 \mathrm{~kg} \mathrm{ha}^{-1}\right)$ registered highest seed yield followed by Pl 6 (1377.4 $\left.\mathrm{kg} \mathrm{ha}^{-1}\right)$ and Subrata $\left(1319.8 \mathrm{~kg} \mathrm{ha}^{-1}\right)$. In second year Subrata $\left(1301.8 \mathrm{~kg} \mathrm{ha}^{-1}\right)$ recorded highest yield followed by Moitri (1191.5 kg ha ${ }^{-1}$ ) and PL 6 $\left(1174 \mathrm{~kg} \mathrm{ha}^{-1}\right)$. Pooled analysis showed that Moitri (1355.5 kg ha ${ }^{-1}$ ) gave maximum yield followed by Subrata $\left(1310.1 \mathrm{~kg} \mathrm{ha}^{-1}\right)$ and the lowest yield was recorded in PL $6(1276.1 \mathrm{~kg}$ $\mathrm{ha}^{-1}$ ) (Table 3a). No significant differences did exist between Moitri and Subrata in terms of branch plant ${ }^{-1}$, pod plant ${ }^{-1}$, plant population $\mathrm{m}^{-2}$, dry matter accumulation, 1000 seed weight and seed yield. According to Gupta and Bhowmick (2005) seed yield of lentil under relay cropping with long duration rice was $1708 \mathrm{kgha}^{-1}$ in Subrata, $1642 \mathrm{~kg} \mathrm{ha}^{-1}$ in WBL 77 and $1366 \mathrm{~kg} \mathrm{ha}^{-1}$ in B 77 cultivars. It is interesting to note that the seed yield was considerably lower in the second year. The percent reduction in yield in the second year for PL 6, Moitri and Subrata were 14.7, 21.6 and 1.3 respectively. This was probably due to the lower plant populations in the second year. Attiya (1985) observed that the faba bean seed yield increased with increasing plant population. Similar report was found Ayaz et al., (1999) for chickpea, lentil, lupins and pea. Das (1992) reported that lentil variety B77 is suitable for relay cropping in West Bengal.

Fertilizer levels significantly affected the seed yield of the varieties irrespective of cultivars. F3 (1670.4 $\mathrm{kg} \mathrm{ha}^{-1}$ in first year, $1448.0 \mathrm{~kg} \mathrm{ha}^{-1}$ in second year and $1559.2 \mathrm{~kg} \mathrm{ha}^{-1}$ in pooled analysis) fertilizer levels recorded significantly higher seed yield in both the years followed by F1 fertilizer levels (1467.9 $\mathrm{kg} \mathrm{ha}^{-1}$ in first year, $1328.2 \mathrm{~kg} \mathrm{ha}^{-1}$ in second year and $1398.0 \mathrm{~kg} \mathrm{ha}^{-1}$ in pooled analysis) (Table 3a). Percentage increase in the seed yield under F1, F2 and F3 fertilizer levels were 38.9, 28.4 and 54.9respectively over control. Ayaz (2001) reported that, positive, linear and significant relationships exist between seed yield and total dry matter of grain legumes.

Fertilizer levels significantly altered the seed yield of the varieties. In first year PL 6 under F1 fertilizer combination (1722.0) $\mathrm{kg} \mathrm{ha}^{-1}$ recorded highest yield. The V1F3, V1F1 (1545.2 kg ha $\left.{ }^{-1}\right)$, V2F2 (1578.7 $\left.\mathrm{kg} \mathrm{ha}^{-1}\right)$, V2F3 (1697.7 kg ha ${ }^{-1}$ ) and V3F3 (1591.7 kg $\mathrm{ha}^{-1}$ ) treatment combination were statistically at par. Moitri under F3 fertilizer combinations $\left(1509 \mathrm{~kg} \mathrm{ha}^{-1}\right)$ gave maximum seed yield in second year. No significant variation was observed between Moitri and Subrata (1444.9 $\mathrm{kg} \mathrm{ha}{ }^{-1}$ ) under F3 fertilizer combination. Pooled result showed that, Moitri recorded highest seed yield under F3 fertilizer combination $\left(1603.8 \mathrm{~kg} \mathrm{ha}^{-1}\right)$. Moitri, PL 6 $\left(1555.6 \mathrm{~kg} \mathrm{ha}^{-1}\right)$ and Subrata (1518.3 $\left.\mathrm{kg} \mathrm{ha}^{-1}\right)$ were statistically at par under F3 fertilizer combination (Table 3b). All three cultivars performed better under F3 fertilizer combination. 
After two years of experimentation it was concluded that cultivar Moitri and $20 \mathrm{~kg} \mathrm{~N}$, $40 \mathrm{~kg} \mathrm{P}_{2} \mathrm{O}_{5}$ and $40 \mathrm{~kg} \mathrm{~K} \mathrm{O}_{2} \mathrm{O}$ ha- 1 at basal $+2 \%$ Urea foliar spray at 45 DAS and 65 DAS can be adopted in relay cropping of lentil with long duration rice in New Alluvial Zone of West Bengal for higher grain yield. In case of non-availability of Cultivar Moitri we can adopt Subrata cultivar with same fertilizer level.

\section{References}

Abou-El-Nour, E.A.A., 2002. Can supplemented potassium foliar feeding reduce the recommended soil potassium? Pak. J. Biol.Sci.5 (3): 25926.

Ali, Md. O., Zuberi, M. I. and Sarker, A. 2012. Lentil relay cropping in the ricebased cropping system: An innovative technology for lentil production, sustainability and nutritional security in changing climate of Bangladesh. Journal of Food Science Engineering, 2: 58 .

Attiya, H.J., 1985. The effects of plant population, growth regulators and irrigation on development and yield of spring sown field beans (Vicia faba L.) (Doctoral dissertation, Lincoln College, University of Canterbury).

Ayaz, S., 2001. Variability of harvest index in four grain legume species (Doctoral dissertation, Lincoln University).

Ayaz, Shaukat, B. A., McKenzie, and G. D. Hill. 1999. "The effect of plant population on dry matter accumulation, yield and yield components of four grain legumes." Interaction 105.5.1, 45.

Bhowmick, M.K., 2008. Effect of foliar nutrition and basal fertilization in lentil under rainfed conditions. Journal of Food Legumes. 21(2): 115-116.

Das, N. R., Datta, B. 1992. Grain yields of rainfed rice-lentil as affected by $\mathrm{N}$ fertilizer and biofertilizer. InternationalRice-Research-Newsletter. 17(2): 20.

De Almeida Costa, G.E., da Silva QueirozMonici, K., Pissini Machado Reis, S.M. and de Oliveira, A.C. 2006. Chemical composition, dietary fibre and resistant starch contents of raw and cooked pea, common bean, chickpea and lentil legumes. Food Chemistry 94, 32 7-330.

Dixit, G.P., Katiyar, P.K., Singh, B.B. and Kumar, S. 2009. Lentil varieties in India, Technical Bulletin, Indian Institute of Pulses Research, Kanpur, pp. 13.

El-Fouly, M.M., and El- Sayed, A.A. 1997. Foliar fertilizer: An environmentally friendly application of fertilizers. Dahlia Greidinger International symptosium on "Fertilizer and Environment" 24-27 March, Haifa, Israel, Ed. John, J. 346357.

Gupta, S., and Bhowmick, M. K. 2005. Scope of growing lathyrus and lentil in relay cropping systems after rice in West Bengal, India. Centre for Legumes in Mediterranean Agriculture (CLIMA), Crawley, Australia, Lathyrus Lathyrism Newsletter, 4, pp 2833, 14 ref.

Kochhar, P.L., and Krishnamoorthy, H.N. K. 1988. A Text Book of Plant Physiology (pp: 174,175,165-168,179,180).

Kuttimani, R., 2012. Response of lentil to foliar fertilization of phosphate. New Agriculturist2 (1): 85-86.

Martens, J. R. T., Hoeppner, J. W. and Entz, M. H. 2001. Legume cover crops with winter cereals in Southern Manitoba: establishment, productivity, and microclimate effects. AgronomyJournal. 93(5): 1086-1096.

Parya, M., Dutta, S. K., Jena, S., Nath, R. and Chakraborty, P.K. 2010. Effect of thermal stress on wheat productivity in West Bengal. Journal of Agrometerology, 12(2): 217-220. 
Roy, A., Aich, S.S., Bhowmick, M.K. and Biswas, P.K. 2009. Response of lentil varieties to sowing time in plains of West Bengal. Journal of crop and weed, 5 (2): 92-94.

Salisbury, F.B., and C.W. Ross. 1985. Plant physiology, $3^{\text {rd }}$ ed. Wadsworth, Belmont, CA. 540 p. Science 102 (6): 874-881.

Sharma, R.K., Bhoi, S.K., Pandey, N., Shinde, S. and Pandey, V.K., (2014), Agriculture at a glance, ASTRAL, pp. 31.

Smolen, S., And W. Sady. 2008. Effect of various nitrogen fertilization and foliar nutrition regimes on carrot (Daucus carota L.). Yield. J. Hort. Sci. Biotechnol., 83: 427-434

Smolen, S., and W. Sady. 2009. Effect of various nitrogen fertilization and foliar nutrition regimes on the concentrations of sugars, carotenoids and phenolic compounds in carrot (Daucus carota L.). Scientia Horticulturae, 120: 315324.

Williams, P. C., and Singh, U. 1988. Quality screening and evaluation in pulse breeding. p. 445-457. In: $\mathrm{R} \quad \mathrm{J}$ Summerfield (ed.), World Crops: Cool Season Food Legumes. Kluwer Academic Publishers, Dordrecht. The Netherlands.

Zayed, B.A., Salem, A. K. M. and El Sharkawy, H. M. 2011. Effect of different micronutrients trearments on rice (Oryza sativa L.) growth and yield under saline soil conditions. World $J$. Agric. Sci., 7(2): 179-184.

\section{How to cite this article:}

Kundu, M.K., Sukanta Das, Sanjib Kumar Mandi and Rajib Nath. 2017. Effect of Fertilizer Levels on Growth Attributes and Seed Yield of Lentil Varieties under Relay Cropping with Long Duration Rice in New Alluvial Zone of West Bengal. Int.J.Curr.Microbiol.App.Sci. 6(9): 466-478. doi: https://doi.org/10.20546/ijcmas.2017.609.056 